\title{
An Experimental Image Guided Surgery Simulator for Hemicricolaryngectomy and Reconstruction by Tracheal Autotransplantation
}

Filip Schutyser ${ }^{1}$, Johan Van Cleynenbreugel ${ }^{1}$, Vincent Vander Poorten ${ }^{2}$, Pierre Delaere $^{2}$, Guy Marchal ${ }^{1}$, and Paul Suetens ${ }^{1}$

1 Laboratory for Medical Image Computing (ESAT and Radiology) K.U.Leuven,

${ }^{2}$ Departement of Oto-Rhino-Laryngology Head and Neck Surgery University Hospitals of Leuven, Herestraat 49, B-3000 Leuven, Belgium

Filip.Schutyser@uz.kuleuven . ac.be

\begin{abstract}
In this paper we describe the current state of our surgical planning environment for a conservation method for unilateral glottic cancer with significant subglottic extension. The latter consists of a hemicricolaryngectomy and subsequent reconstruction of the laryngeal defect with a transferable patch of revascularized cervical trachea.

In order to restore the three crucial functions of the larynx: airway patency, speech and swallowing, such a tracheal patch has to meet a typical surgical constraint (so-called paramedian position at glottic level). As such constraints are difficult to realise intraoperatively, we designed an image-based surgery simulator for the application. The planning environment visualizes medical image volumes (3D surfaces of the anatomy of interest together with multiplanar reslices) and takes specific surgical constraints into account. The guidance of the surgeon through the planning is menu-driven. To partly test our hypothesis that postoperative morphological results can be optimized by $3 \mathrm{D}$ planning, a cadaver study was set up. We report on both the planning environment and on the results of this study.
\end{abstract}

Keywords Image guided therapy, surgery simulation, hemilaryngectomy, tracheal autograft

\section{Introduction}

Current surgical treatment for unilateral glottic cancer with significant subglottic extension is a total laryngectomy. For these indications, a conservation procedure was developed by the clinical partners involved in this research $[1,2]$. The technique consists of a hemicricolaryngectomy and subsequent reconstruction of the laryngeal defect with a transferable patch of revascularized cervical trachea. The advantage of this technique over the formerly performed total laryngectomy is the absence of a permanent tracheostoma and the preservation of the voice, obviating the need for a voice prosthesis and its accompanying inconveniences. 
In this conservation procedure, the position of the patch at the glottic level is pivotal in determining the functional result of the technique with regard to airway patency, speech and swallowing.

We hypothesize that the postoperative morphological results of this technique can be optimized if the procedure is planned in 3D, starting from the CT-images that are routinely made in the diagnostic workup of such patients. A crucial step towards this hypothesis is the ability to respect the constraints on the patch at the glottic level. For this purpose we have developed an image guided surgery simulator. In it, the dimensions and location of the laryngeal resection and of the tracheal patch are planned, taking into account a number of surgical constraints, the one at the glottic level being the most important. From this simulation, actual measurements are obtained of the specimen to be resected and of the patch to be used for the reconstruction. To test that crucial step towards our hypothesis, a cadaver study was set up.

In section 2 some background information is given on the surgical technique. Section 3 outlines our approach towards surgery simulation. The cadaver experiments and their results are described in section 4 . Concluding remarks finish the paper in 5 .

\section{Background}

The surgeons involved in this research $[1,2]$ have postulated the paramedian position to be the ideal patch position to provide for the three crucial larynx functions. If the patch is positioned in this way, the reconstructed "larynx" would resemble a situation of one paralyzed and one intact vocal fold at the glottic level, a situation known to give acceptable functional results. This position is however difficult to achieve intraoperatively because variable tumor dimensions in patients with variable laryngeal dimensions demand an individualized resection and an individually tailored tracheal patch for reconstruction.

So far (July 1998) fourteen patients have undergone this operation, the resection and the reconstruction being based on the clinical intuition of the surgeons. All patients did well postoperatively, but varying results with respect to airway patency, speech and swallowing have been observed, the results being better with the definitive patch position being closer to the paramedian position. In order to optimize the postoperative functional results of this new surgical technique, image guided surgical planning was envisaged.

\section{Simulation environment}

At the core of this simulation environment is the notion of representing the 3D space covered by the medical imaging data volume(s) as a 3D scene. In that scene multiplanar reslices are co-presented with image derived 3D surface triangle mesh representations of the anatomical structures of interest. The concepts of our environment are described in [3] and are currently implemented on top of OpenInventor [4]. As a working hypothesis, we assume that the surfaces 

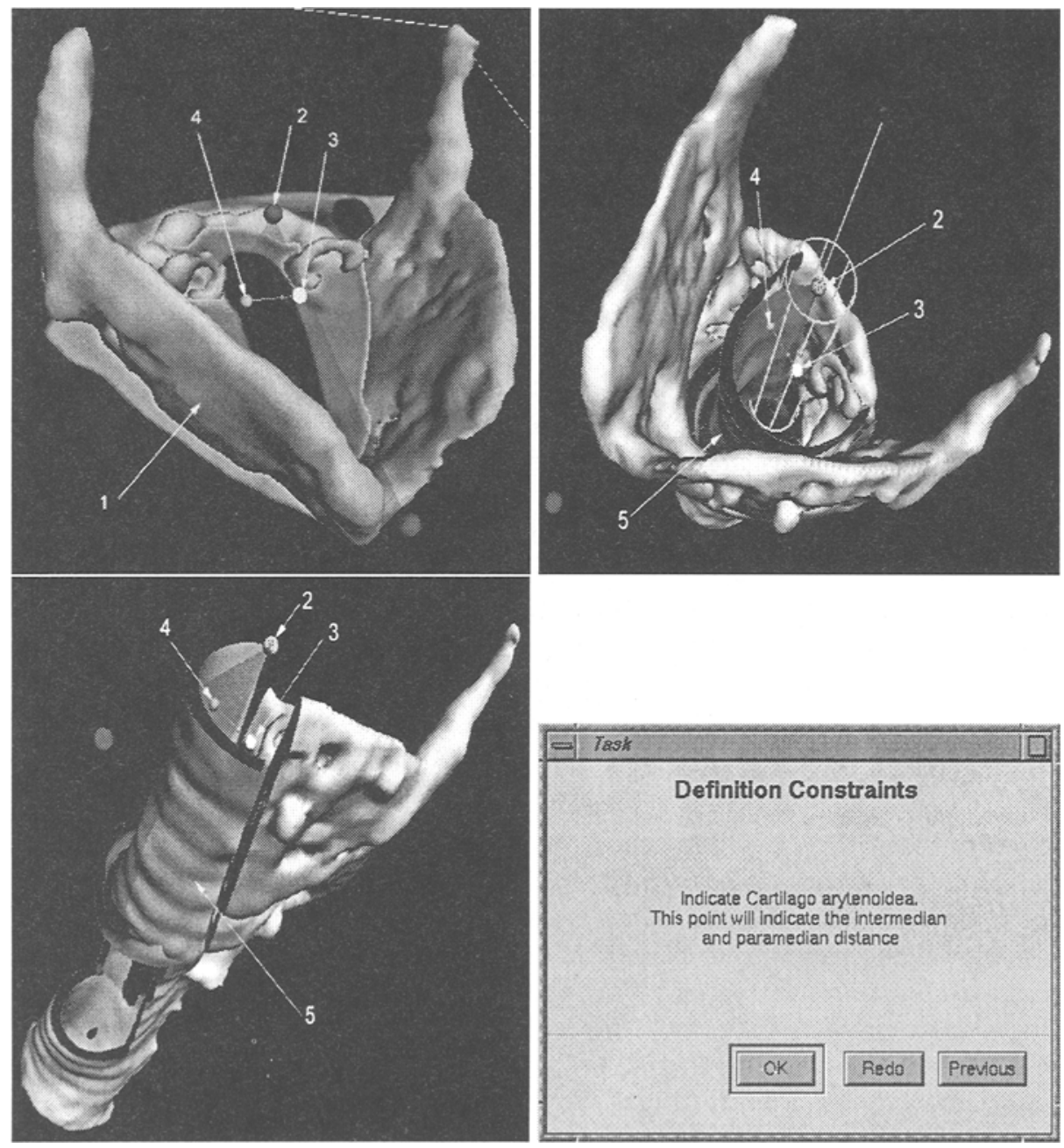

Fig. 1. This figure illustrates our laryngectomy simulation applied to a cadaver larynx. In the upper left part, an axial CT slice at subglottic level and a surface model of the larynx (label 1) are partially shown. Label 2 refers to one of the 3D points defining the symmetry plane of the larynx. Label 3 refers to a 3D point added by the surgeon on an anatomical landmark on the CT slice at glottic level. From this point and from the position of the symmetry plane, the simulator calculates the point labeled by 4 . This is the point at paramedian position. In the upper right part, the same structures are labeled and the tracheal patch (label 5) has already been cut and translated to an axis in the symmetry plane through point 2 . A manipulator is attached to the patch in order to let the surgeon rotate it towards the paramedian position (4). This result is shown in the lower left part, where the patch (5) is rotated in such a way that it makes contact with point 4 . At that moment the patch and the larynx are resected as shown. The lower right part shows a typical example of a menu. This menu asks the surgeon to indicate the cartilago arytenoidea (3). 
of anatomical structures are rigid. To simulate resecting actions, cutting algorithms (cutting with a plane, cutting along a free-hand drawn line) on surface representations are developed $[5,6]$. The basis of these cutting algorithms are classification of triangles and retriangulation along the intersection line.

From the routinely made spiral CT images, 3D surfaces of the larynx and trachea are derived. For real patients, the vicinity of other structures and the large differences in degree of calcification of the larynx, necessitates manual segmentation. Structures like the arythenoids which are hard to see on CT, need extra attention. However, for cadaver larynges, all segmentation can be done by thresholding. All the cadaver larynges used for the study discussed in section 4 , are segmented by thresholding.

Constraints typical for success in this new surgical treatment are incorporated in the surgical simulator. The paramedian position is the constraint defining the desirable morphological result. Other constraints define the cutting planes: the position of the radial forearm fascial flap revascularizing the cervical trachea segment, the middle of the cartilago thyroidea, ... These constraints determine different important surgical parameters (e.g. the distance between the upper border of the arythenoid and the lower border of the cricoid determines the cranio-caudal length of the tracheal patch). Based on these constraints, the repositioning of the tracheal patch is determined. These constraints are visualised as geometric entities such as points, planes, axes, allowable transformations, and can be attached as annotations to particular locations of the 3D scene.

To complete the simulation, raw basic tools (cutting objects, annotation tools, ...) alone do not suffice. To supply extra guidance to the surgeon, a menu-based approach pilots the surgeon through a number of subtasks to complete the virtual operation. These subtasks ask the surgeon to instantiate the constraints on the individual anatomy of the patient. Based on his expertise, he indicates a possible configuration for the constraints on the CT-images or the 3D surface representation. When following these subtasks, cutting actions are performed. Once he finishes a first simulation, he can redo some subtasks to optimize some of the previous choices. In figure 1, details of this simulation are pointed out.

Once an optimal configuration is selected, a set of measurements easily reproducible during the actual operation is derived. Measurement tools resembling tools common to the surgeon in the operating theatre are available, e.g. (flexi)rulers.

\section{Experiments}

To test the crucial step towards our hypothesis mentioned in the introduction, a cadaver study was set up. We tested (on seven cadaver larynges) whether the simulated operation - i.e. the optimal one according to the postulate of the surgeons and thus taking into account the defined constraints and the anatomy of the larynx - could be advantageously employed during actual operation. Therefore post-operative imaging was used to measure how the constraints were met. 
To enable post-to-pre registration fiducial markers were attached to the cadaver larynges.

For each cadaver larynx, pre-operative CT imaging was used to plan the operation. The surgeon repeated and adapted the planning until an optimal configuration was reached. Based on this configuration, enough reproducible distances (different measurements on the larynx: length of the tracheal patch, internal "circular" distances on the tracheal patch, see figure 2) were measured to reconstruct the virtual operation during the actual surgery.

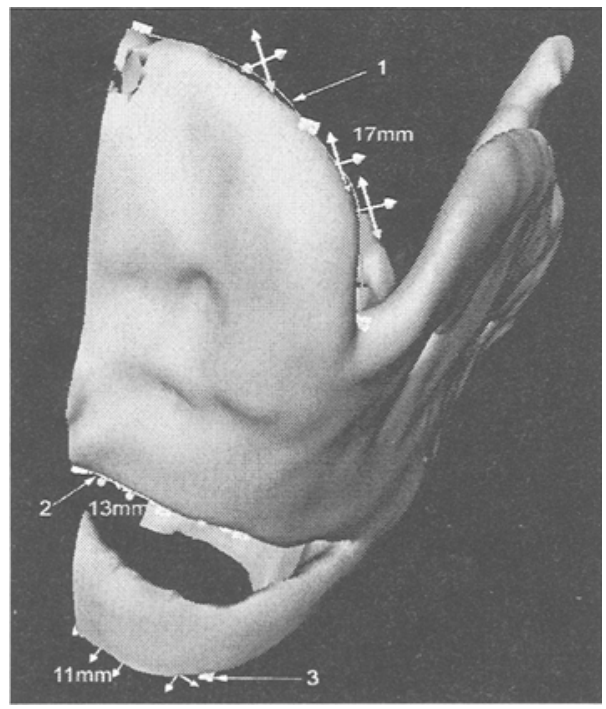

Fig. 2. This figure shows the measurements on the part of the larynx that will be resected. All measurements start from the middle of the larynx, a landmark which can be easily determined intra-operatively. Label 1 and 2 point to distances on the cartilago thyroidea, label 3 points to a distance on the arcus cartilago cricoidea. The measurement tool is a flexiruler (i.e. a planar, but curved distance).

After the actual surgery, post-operative CT imaging of the resected larynges makes it possible to compare the planned and actual results. The post-to-pre registration was done by least squares point based matching on the fiducial markers. After registration, the post-operative images are resampled on the grid of the pre-operative image volume to generate comparable measurements. To apply adequate measurements, magnitudes (see figure 3 ) presumed to be related to the functional results of the operation were chosen [1].

Based on the seven test cases, a few trends become clear. First of all, the major purpose of the planning, i.e. assuring that the patch meets the paramedian position, is reached. All the tracheal patches were positioned conforming to the paramedian position. However, concerning the anterior-posterior glottic diameter 


\begin{tabular}{|c|c|c|c|c|c|c|c|}
\hline Larynx Nr & $\begin{array}{c}\text { AP simul } \\
\mathrm{mm}\end{array}$ & $\begin{array}{c}\text { AP post } \\
\mathrm{mm}\end{array}$ & $\begin{array}{c}\text { GL simul } \\
\mathrm{mm}^{2}\end{array}$ & $\begin{array}{c}\text { GL post } \\
\mathrm{mm}^{2}\end{array}$ & $\begin{array}{c}\text { SG simul } \\
\mathrm{mm}^{2}\end{array}$ & $\begin{array}{c}\text { SG post } \\
\mathrm{mm}^{2}\end{array}$ & $\begin{array}{c}\Delta \text { PM } \\
\mathrm{mm}\end{array}$ \\
\hline 1 & 15 & 17 & 104 & 90 & 125 & 110 & -1 \\
2 & 20 & 17 & 126 & 111 & 170 & 129 & $\mathbf{0}$ \\
3 & 13 & 16 & 52 & 53 & 178 & 213 & 0 \\
4 & 21 & 21 & 95 & 55 & 191 & 121 & -2 \\
5 & 17 & 20 & 77 & 116 & 89 & 143 & 1 \\
6 & 20 & 22 & 80 & 109 & 149 & 157 & 0 \\
7 & 19 & 14 & 64 & 45 & 105 & 115 & $\mathbf{0}$ \\
\hline
\end{tabular}

Table 1. Measurements on seven test larynges.

$\mathrm{AP} \quad=$ anterior-posterior diameter at glottic level

$\mathrm{GL}=$ area of airway lumen at glottic level

$\mathrm{SG}=$ area of airway lumen at subglottic level

$\Delta \mathrm{PM}=$ difference between simulated paramedian distance and postoperative patch position (negative distance means that the patch is more proximal).
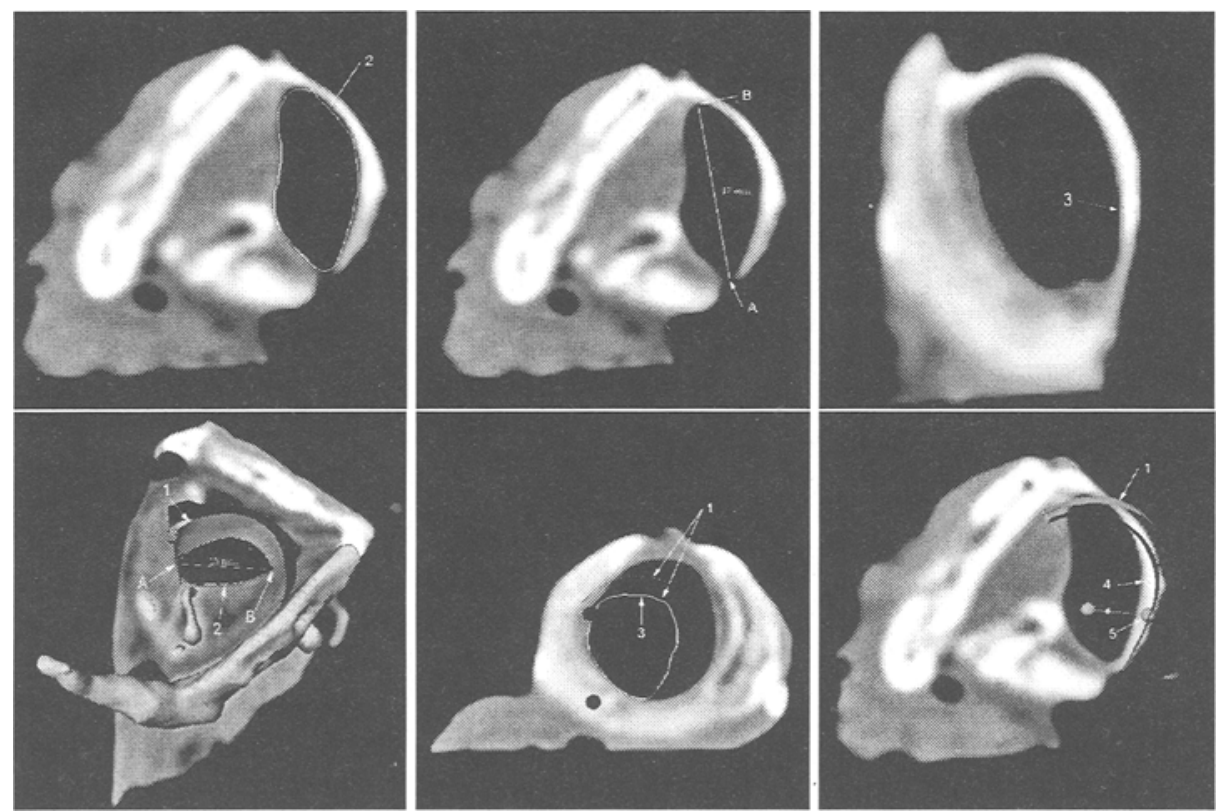

Fig. 3. This figure illustrates, for the particular case of larynx 1, the quantitative measures used in table 1 . The upper row shows three slices through the post-operative CT volume. The lower row shows two (left, middle) slices through the pre-operative and one (right) through the post-operative $\mathrm{CT}$ volume, in combination with simulation data. Label 1 indicates the planned tracheal patch. Line AB, label 2 and label 3 refer to the anterior-posterior glottic diameter, the area of the airway lumen at glottic level and the area of the airway lumen at subglottic level respectively. Label 4 points to the actual tracheal patch. Label 5 points to the paramedian position. This position is shared by both the planned and the actual patch. 
and the airway lumen areas (at glottic level and subglottic level), there are fairly small differences (see Table 1). The fact there is a slight variation in the stitches used to fix the patch to the cricoidal remnant posteriorly and to the vocal cord remnant anteriorly, can explain the differences from the planned configuration. Moreover, in some cases the tracheal patch is stretched in sagittal direction, a situation we will try to foresee in the future.

A more rigorous validation in the near future will investigate the importance of deformable patches in the planning system and the methods of attaching the tracheal patch and the vocal cord. We also consider more advanced methods (video matching) for intra-operative support than a list of measurements.

\section{Discussion}

When following our approach from planning to surgery, even when resecting cadaver larynges, surgeons report to feel more comfortable, mainly because they have a higher pre-operative certainty of meeting the paramedian position. Additionally they report to obtain a better insight in the particular anatomy and even in sometimes competing constraints, they are only implicitly aware of (e.g. reaching the paramedian position at glottic level versus minimal decrease of airway lumen area at subglottic level).

During the course of the design of the planning system and its use in the cadaver experiments, the surgeons also reported to gain insight with respect to refinement of their technique. Furthermore, the system has also been applied to three patients at this moment (see figure 4). From the experiences gained by this actual use of the laryngectomy simulator, we were forced to reconsider our basic assumptions.

Earlier, we assumed that our simulation environment only had to handle rigid anatomical structures. Rigidity certainly is a property of the larynx. However, for the tracheal patch, this assumption is not always valid. Although the surgery itself can be performed by using an undeformed patch, this way of working can lead to too large a resection. Only in the case where a maximally possible resection is really needed - e.g. for a tumor including the anterior coomissura and crossing the larynx symmetry plane -, a rigid patch would be appropriate. As this is not always needed in clinical practice, future work will investigate the application of deformation techniques turning the rigid tracheal patch into a flexible one.

\section{Acknowledgments}

This work is performed, partly under the Flemish government-funded IWT ITAGBO202, TeleVisie project, and partly under the EU-funded Brite Euram III PISA project (nr. BRPR CT97 0378). Partners in the latter are Materialise NV, Belgium; Philips Medical Systems BV, the Netherlands: ICS-AD; DePuy International Ltd, UK; Ceka NV, Belgium; K.U. Leuven, Belgium: ESAT/Radiology \& Div. Biomechanics; University of Leeds, UK: Research School of Medicine. 

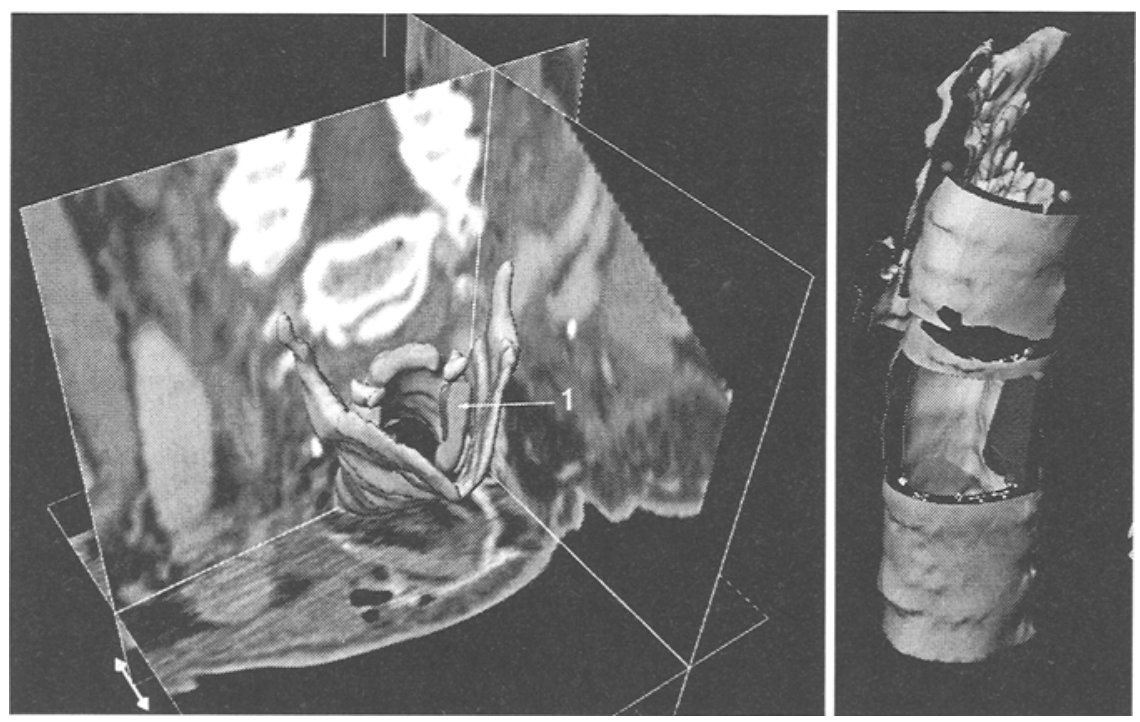

Fig. 4. This figure illustrates the simulator applied to a real patient. On the left, a 3D scene representation of a larynx containing a T3 glottic cancer with vocal fold fixation (label 1) is shown. On the right, the result of laryngeal resection and tracheal patch relocation is depicted.

\section{References}

1. P.R. Delaere, V. Vander Poorten, A. Goeleven, M. Feron, R. Hermans: Tracheal Autotransplantation: a Reliable Reconstructive Technique for Extended Hemilaryngectomy Defects. Laryngoscope 108: June 1998, p 929 - 934

2. P.R. Delaere, V. Vander Poorten, R. Hermans: Autotransplantation of the trachea: experimental evaluation of a reconstructive technique for extended hemilaryngectomy defects. accepted for publication in Annals of Otology, Rhinology and Laryngology, 1998

3. J. Van Cleynenbreugel, K. Verstreken, G. Marchal, P. Suetens: A flexible environment for image guided virtual surgery planning. Proc. VBC96, Lect. Notes in Computer Science, 1131, 1996, p 501-510

4. J. Werneke: The Inventor Mentor, Programming Object-Oriented 3D Graphics with OpenInventor Addison-Wesley, release 2, 1994

5. F. Schutyser, J. Van Cleynenbreugel, G. Marchal, P. Suetens: An interactive environment for constraint-based pre-operative simulation of rigid tomia procedures. Technical Report KUL/ESAT/PSI/9801, February 1998

6. F. Schutyser, J. Van Cleynenbreugel, G. Marchal, P. Suetens: Implementation of Tomia algorithms on Triangle Strip Sets. Technical Report KUL/ESAT/PSI/9802, February 1998 